a well-defined extent. As jamming phenomena can be observed in diverse systems from highway traffic to foams to glasses, the possibility exists that such a length scale has more general implications that extend well beyond a finger being pushed through the sand.

Matthew B. Stone, David P. Bernstein,

Rachel Barry, Matthew D. Pelc, Yee-Kin Tsui, Peter Schiffer

Department of Physics and Materials Research Institute, Pennsylvania State University, University Park, Pennsylvania 16802, USA

e-mail: schiffer@phys.psu.edu

1. Albert, R., Pfeifer, M. A., Barabási, A.-L. \& Schiffer, P. Phys. Rev. Lett. 82, 205-208 (1999).

2. Yu, H. S. \& Mitchell, J. K. J. Geotech. Geoenvir. Eng. 124, 140-149 (1998).

3. Peterson, R. W. in Calibration Chamber Testing: PTProc. First Intl Symp. Calibration Chamber Testing (ed. Huang, A.-B.) 315-328 (Elsevier, New York 1991).

4. Vanel, L. \& Clément, E. Eur. Phys. J. B 11, 525-533 (1999).

5. Geng, J. et al. Phys. Rev. Lett. 87, 035506-035509 (2001).

6. Liu, C.-H. et al. Science 269, 513-515 (1995).

7. Da Silva, M. \& Rajchenbach, J. Nature 406, 708-710 (2000).

8. Reydellet, G. \& Clément, E. Phys. Rev. Lett. 86, 3308-3311 (2001).

9. Bouchaud, J. P., Cates, M. E. \& Claudin, P. J. Physique I 5, 639-656 (1995).

10. Mueggenburg, N. W., Jaeger, H. M. \& Nagel, S. R. Phys. Rev. E 66, 031304-031312 (2002).

11. Liu, A. J. \& Nagel, S. R. Nature 396, 21-22 (1998).

12. O’Hern, C. S., Langer, S. A., Liu, A. J. \& Nagel, S. R. Phys. Rev. Lett. 86, 111-114 (2001).

13. Vergeles, M., Keblinski, P., Koplik, J. \& Banavar, J. R. Phys. Rev. Lett. 75, 232-235 (1995).

Supplementary information accompanies this communication on Nature's website.

Competing financial interests: declared none.

\section{Transgenic mice}

\section{Fat-1 mice convert $n-6$ to $n-3$ fatty acids}

$\mathrm{M}$ ammals cannot naturally produce omega-3 (n-3) fatty acids - beneficial nutrients found mainly in fish oil - from the more abundant omega-6 (n-6) fatty acids and so they must rely on a dietary supply ${ }^{1}$. Here we show that mice engineered to carry a fat-1 gene from the roundworm Caenorhabditis elegans can add a double bond into an unsaturated fatty-acid hydrocarbon chain and convert $n-6$ to $n-3$ fatty acids. This results in an abundance of $n-3$ and a reduction in $n-6$ fatty acids in the organs and tissues of these mice, in the absence of dietary $n-3$. As well as presenting an opportunity to investigate the roles played by $n-3$ fatty acids in the body, our discovery indicates that this technology might be adapted to enrich $n-3$ fatty acids in animal products such as meat, milk and eggs.

The fat- 1 gene of $C$. elegans encodes an $n-3$ fatty-acid desaturase enzyme that converts $n-6$ to $n-3$ fatty acids and which is absent in most animals, including mammals $^{2,3}$. We transferred this fat-1 gene into mice and raised them alongside wild-type mice maintained on an identical diet that was high in $n-6$ but deficient in $n$ - 3 fatty acids. However, the fatty-acid profiles of the two groups turned out to be quite different (Fig. 1). The tissues of wild-type animals contain polyunsaturated fatty acids that are mainly (about 98\%) n-6 linoleic acid (designated an 18:2n-6 fatty acid as it has 18 carbon atoms and 2 double bonds, one at position $n-6)$ and arachidonic acid (AA, 20:4 n-6), with very little $n-3$ fatty acid (from a dietary source). By contrast, the transgenic animal tissues are rich in $n-3$ polyunsaturated fatty acids, including linolenic acid (18:3n-3), eicosa-pentaenoic acid (EPA, 20:5 n-3), docosa-pentaenoic acid (DPA, 22:5n-3) and docosahexaenoic acid (DHA, 22:6n-3).

The concentrations of $n-6$ linoleic and arachidonic acids in the tissues of the transgenic mice are significantly reduced, indicating that $n-6$ fatty acids have been converted to $n-3$, causing the ratio of $n-6$ to $n-3$ to drop from $20-50$ to almost 1 . This $n$ - 3 enrichment at the expense of $n-6$ gives a balanced ratio of $n-6$ to $n-3$ and of AA/(EPA+DPA+DHA) in all of the organs and tissues tested (Table 1). Transgenic skeletal muscle contains more EPA than DHA, but DHA is the dominant $n$-3 fatty acid in other organs.

We have examined the tissue fatty-acid profiles in four generations of transgenic mouse lines (homozygote or heterozygote) and find consistently raised $n$ - 3 fatty acids, indicating that the transgene is functionally active in vivo and transmittable. The transgenic mice appear to be normal and healthy.

Efforts have been made to incorporate $n-3$ fatty acids into the food supply ${ }^{1,4}$ because of their health benefits and concern over the high $n-6: n-3$ ratio in Western diets. Our findings suggest a new strategy for producing food that is enriched in $n-3$ fatty acids from livestock carrying an $n-3$ desaturase trans-gene. At present, farm animals are fed fishmeal and other marine products, but this is time-consuming and costly, and is limited by the quantity of the source ${ }^{5}$. Production of $n-3$ fatty acids by the animals themselves would be a cost-effective and sustainable way of meeting the increasing demand; the ideal $n-6: n-3$ ratio of about 1 could be achieved by consuming foods

\begin{tabular}{|c|c|c|c|c|}
\hline \multicolumn{5}{|c|}{ Table 1 Fatty-acid ratios in WT and fat-1 mice } \\
\hline & \multicolumn{2}{|c|}{$n-6 / n-3^{\star}$} & \multicolumn{2}{|c|}{$A A /(E P A+D P A+D H A)$} \\
\hline & $W T$ & $T M$ & $W T$ & $T M$ \\
\hline Muscle & 49.0 & 0.7 & 11.3 & 0.4 \\
\hline Milk† & 32.7 & 5.7 & 15.7 & 2.5 \\
\hline Erythrocyte & 46.6 & 2.9 & 27.0 & 1.6 \\
\hline Heart & 22.8 & 1.8 & 14.3 & 0.9 \\
\hline Brain & 3.9 & 0.8 & 3.6 & 0.7 \\
\hline Liver & 26.0 & 2.5 & 12.5 & 0.9 \\
\hline Kidney & 16.5 & 1.7 & 11.9 & 1.2 \\
\hline Lung & 32.3 & 2.2 & 19.8 & 1.2 \\
\hline Spleen & 23.8 & 2.4 & 17.3 & 1.5 \\
\hline
\end{tabular}

Both wild-type (WT) and transgenic (TM) mice were 8-week-old females fed on the diet described in Fig.1. AA, arachidonic acid (20:4 n-6); EPA, eicosapentaenoic acid (20:5 $n-3)$; DPA, docosapentaenoic acid (22:5 n-3); and DHA, docosahexaenoic acid (22:6 $n-3)$.

*The $n-6: n-3$ fatty-acid ratio is given by $(18: 2 n-6+20: 4 n-6+22: 4 n-6$ $+22: 5 n-6):(18: 3 n-3+20: 5 n-3+22: 5 n-3+22: 6 n-3)$.

†The milk was taken from the stomach contents of 5 -day-old neonatal mice born to wild-type or transgenic mothers.

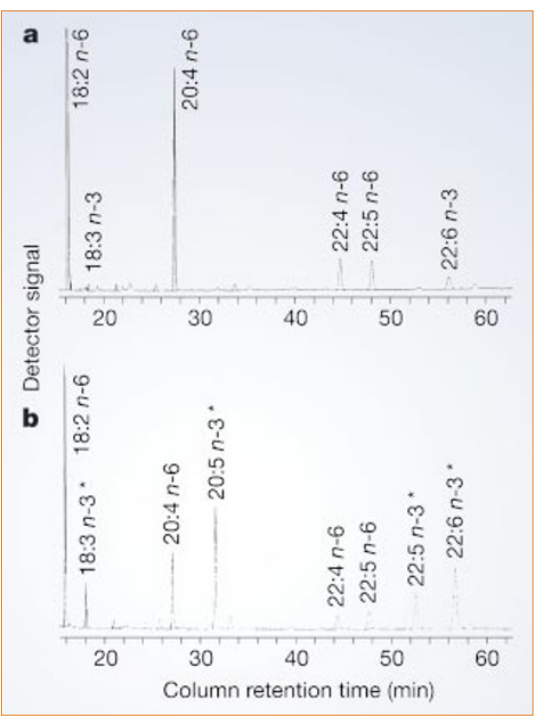

Figure 1 Partial gas chromatograph traces showing the polyunsaturated fatty-acid profiles of total lipid extracted from mouse skeletal muscle. $\mathbf{a}, \mathbf{b}$, Traces from lipid from $\mathbf{a}$, a wild-type mouse, and $\mathbf{b}$, a fat- 1 transgenic mouse (heterozygote). The expression vector used for microinjection contained the humanized fat-1 sequence (with modification of codon usage) and a chicken $\beta$ actin promoter and cytomegalovirus enhancer, which allow high and broad expression of the transgene in mice ${ }^{6,7}$. Both the wildtype and transgenic mice were 8-week-old females that were fed on the same diet, which was high in n-6 but low in n-3 fatty acids. The lipid profiles show that concentrations of $n-6$ polyunsaturated acids (18:2 n-6, 20:4 n-6, 22:4 n-6 and 22:5 n-6) are lower and levels of $n-3$ fatty acids (asterisks) are markedly higher in transgenic (b) than in wild-type (a) muscle. (Homozygotes and heterozygotes have a similar phenotype.)

containing this ratio and without introducing stringent dietary changes.

Our transgenic mice also offer a model for investigating the biological functions of $n-3$ fatty acids and the importance of the ratio of $n-6: n-3$ in disease prevention and treatment. Specific effects of $n-3$ fatty acids and of the $n-6: n-3$ ratio can be tested in different organs and tissues - for example, they may alter gene expression or physiological activity during the life cycle. Our mouse lines could be genetically backcrossed with mouse disease models to test the effects of $n-3$ fatty acids on the pathogenesis and treatment of those diseases.

Jing X. Kang ${ }^{\star}$, Jingdong Wang ${ }^{\star}$, Lin Wu $\dagger$, Zhao B. Kang*

Departments of ${ }^{\star}$ Medicine and $\dagger$ Dermatology, Massachusetts General Hospital and Harvard Medical School, Boston, Massachusetts 02114, USA e-mail:kang.jing@mgh.harvard.edu

\footnotetext{
1. Simopoulos, A. P. et al. (eds) World Rev. Nutr. Diet Vol. 83 (Basel, Karger, 1998)

2. Spychalla, J. P., Kinney, A. J. \& Browse, J. Proc. Natl Acad. Sci. USA 94, 1142-1147 (1997).

3. Kang, Z. B. et al. Proc. Natl Acad. Sci. USA 98, 4050-4054 (2001).

4. Simopoulos, A. P. \& Cleland, L. G. (eds) World Rev. Nutr. Diet Vol. 92 (Basel, Karger, 2003).

5. Naylor, R. L. et al. Nature 405, 1017-1024 (2000).

6. Niwa, H., Yamamura, K. \& Miyazaki, J. Gene 108, 193-199 (1991)

7. Okabe, M. et al. FEBS Lett. 407, 313-319 (1997).

Competing financial interests: declared none.
} 
the standard deviation does not. This is because changing the units corresponds to multiplying the data with a constant $(a)$, which adds a constant to the log values: $\ln a N_{i}=\ln a+\ln N_{i}$. The resulting change in 'synchrony' depends both on $\operatorname{stdev}_{\text {all } i}\left(\ln N_{i}\right)$, which is specific to each year's set of abundances, and on the choice of unit (specifically, $\left.\operatorname{sync}(a N)=\operatorname{sync}(N)+\ln (a) / \operatorname{stdev}_{\text {all }}\left(\ln N_{i}\right)\right)$. Therefore, a change of unit may qualitatively reverse comparisons of synchrony between data sets.

This problem is not resolved by addition of a constant to all log-transformed musk-ox abundances to ensure that these are positive before calculating 'synchrony' (Post and Forchhammer, personal communication), as the relationship between mean $\ln a N$ and stdev $\ln a N$ remains entirely arbitrary. Biological conclusions should not be affected by whether American, metric or other units are used.

Post and Forchhammer's analyses show an apparent tendency for cross-species correlation to decrease with increasing interpopulation distance. However, the few strong correlations describe concurrent trends over decades, rather than the yearto-year variation that was Post and Forchhammer's focus. Plotting cross-species correlation against each $\left(\omega_{\text {musk ox }}^{(i)}, \omega_{\text {caribou }}^{(j)}\right)$ pair shows no consistent pattern. We also correlated growth rates instead of raw abundances and found unsystematic and weak correlations. Although other approaches might be more successful, the data may not be sufficiently precise or relevant to detect the phenomenon if it exists. We conclude that there is currently no proper evidence of climate-induced synchrony between musk oxen and caribou on Greenland.

Jon Olav Vik ${ }^{\star}$, Nils Chr. Stenseth ${ }^{\star}$, Giacomo Tavecchia ${ }^{\star} \dagger$, Atle Mysterud ${ }^{\star}$, Ole Chr. Lingjærde $\neq$

${ }^{*}$ Centre for Ecological and Evolutionary Synthesis, Department of Biology, and $\ddagger$ Department of Informatics, University of Oslo,

PO Box 1050 Blindern, 0316 Oslo, Norway

e-mail:n.c.stenseth@bio.uio.no

$\dagger$ Present address: IMEDA-UIB/CSIC-c Miquel

Marques 21, 07190 Esporles, Spain

1. Moran, P. A. P. Aust. J. Zool. 1, 291-298 (1953).

2. Post, E. \& Forchhammer, M. C. Nature 420, 168-171 (2002).

3. Hurrell, J. W. Science 269, 676-679 (1995).

4. Forchhammer, M. C., Post, E., Stenseth, N. C. \&

Boertmann, D. M. Population Ecol. 44, 113-120 (2002).

Post and Forchhammer reply - Vik et al. question whether we documented spatial synchrony between caribou and musk oxen from Greenland, and whether spatial synchrony within each species related to the North Atlantic Oscillation (NAO) ${ }^{1}$. Attributing spatial synchrony to climate is difficult but possible ${ }^{2}$, and the questions raised by Vik et al. are readily addressed. Contrary to their incorrect statement of our definition of the NAO effect ratio ${ }^{1}$, a strong climatic effect on any pair of populations is not a requisite of climate-induced synchrony. As Moran ${ }^{3}$ argued, and as our analysis illustrated ${ }^{1}$, populations may be synchronized if climate influences each of them similarly, regardless of the magnitude of that influence. Moreover, the standardized NAO effect ratio is associated statistically with the degree of climatic correlation across populations ${ }^{4}$ and hence the degree of synchrony between populations ${ }^{3}$.

As stated previously ${ }^{1}$, we used cross-population covariance $(\mathrm{CV})$ to produce a timeseries index of spatial synchrony, an approach validated in empirical ${ }^{5}$ and theoretical ${ }^{6}$ studies, which have demonstrated the relationship of CV to population synchrony ${ }^{7}$. The simplest test of whether the use of logtransformed data confounds our results is to compare them with results obtained using raw (not log-transformed) data. The correlation between the NAO and $1 / \mathrm{CV}$ of the raw musk-ox data $^{8}(r=-0.57, P=0.001)$ matches exactly the correlation between the NAO and $1 / \mathrm{CV}$ of the log-transformed musk-ox data $(r=-0.59, P=0.001)$. Similarly, results do not vary for caribou, using log-transformed $(r=0.35, \quad P=0.002)$ or raw $(r=0.24$, $P=0.04)$ data. Hence, log-transformation does not influence relationships between the $\mathrm{NAO}$ and spatial synchrony.

Moreover, our results were not influenced by addition of a constant to the logtransformed musk-ox data, which Vik et al. describe as analogous to changing units of abundance. Such a problem would be apparent if the means of the $N_{i}, \ln (N)_{i}$, or $\left[\ln (a)+\ln (N)_{i}\right]$ showed significant and inconsistent correlations with the NAO, but none did $\left(r_{i}\right.$ values of $0.14,0.07$ and 0.07 , respectively; all $P$ values $\geqslant 0.50)$. Vik et al. obtained different results because their direct log-transformation of the decimalform musk-ox data produced negative values, giving statistically invalid $\mathrm{CVs}^{9,10}$. We added 4 to the log-transformed musk-ox data to convert negative values to positives before calculating the $\mathrm{CV}$ precisely to avoid a spurious correlation.

If the NAO-spatial synchrony correlation were, in fact, influenced by the use of log-transformed data, such an artefact should be apparent in two ways, neither of which is discussed by Vik et al. First, the sign of the correlation between the NAO and musk-ox spatial synchrony should change with addition of constants greater than 4 . Second, adding constants to log-transformed caribou data (to which none was originally added ${ }^{1}$ ) should also alter the NAO-caribou synchrony correlation. We checked this by adding constants of up to 10, and in neither case was the correlation altered (Fig. 1).

We conclude that our previous results still stand and that Vik et al. cannot offer a

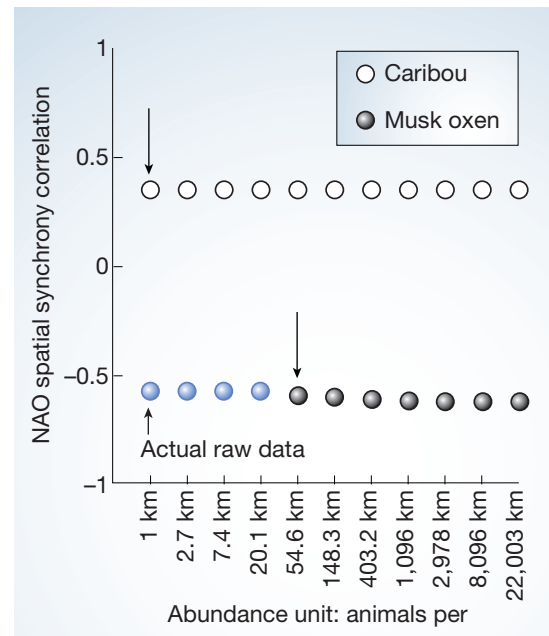

Figure 1 Addition of constants from zero to ten (corresponding to abundance units shown on the $x$-axis) to In-transformed density estimates does not influence the sign of the correlation between the NAO index and spatial synchrony for musk oxen or caribou. Points designated by an arrow are the correlations reported in our original analysis ${ }^{1}$. For the In-transformed musk-ox data, addition of constants less than four, as done by Vik et al., produces spurious correlations because the resulting negative values do not give statistically valid coefficients of variation ${ }^{9,10}$. Thus, the blue points indicate the correlations obtained when using the raw musk-ox data ${ }^{8}$ with constants of zero to three added; note that the point denoted as raw data in Fig. 1 of Vik et al. is in fact a direct Intransformation of the actual raw data.

means of analysing or an alternative explanation for spatial synchrony within and across these species.

\section{Eric Post ${ }^{\star}$, Mads C. Forchhammer $\dagger$}

${ }^{*}$ Department of Biology, The Pennsylvania State University, 208 Mueller Lab, University Park, Pennsylvania 16802, USA

e-mail: esp10@psu.edu

$\dagger$ Department of Population Biology, Biological Institute, University of Copenhagen, 2100

Copenhagen, Denmark

1. Post, E. \& Forchhammer, M. C. Nature 420, 168-171 (2002)

2. Cattadori, I. M., Merler, S., \& Hudson, P. J. J. Anim. Ecol. 69, 620-638 (2000).

3. Moran, P. A. P. Aust. J. Zool. 1, 291-298 (1953).

4. Sokal, R. R. \& Rohlf, F. J. Biometry: The Principles and Practice of Statistics in Biological Research (Freeman, New York, 1995).

5. Holyoak, M. \& Lawler, S. P. J. Anim. Ecol. 65, 640-652 (1996).

6. Ims, R. A. \& Steen, H. Oikos 57, 381-387 (1990).

7. Buonaccorsi, J. P. et al. J. Theor. Biol. 224, 107-114 (2003).

8. Forchhammer, M. C. \& Boertmann, D. M. Ecography 16, 299-308 (1993).

9. Koenig, W. D. \& Knopps, J. M. H. Am. Nat. 155, 59-69 (2000).

10. Wallis, W. A. \& Roberts, H. V. Statistics: A New Approach (Free, New York, 1956).

\section{corrigendum}

Fat-1 mice convert $\boldsymbol{n}-\mathbf{6}$ to $\boldsymbol{n}-\mathbf{3}$ fatty acids

Jing X. Kang, Jingdong Wang, Lin Wu, Zhao B. Kang Nature 427, 504 (2004)

J. X. K. is the inventor and co-applicant (with Massachusetts General Hospital, a non-profit organization) of a patent application relevant to this work (USSN 60/275,222: W002072028), which should therefore have been declared as a competing financial interest. 